\title{
FPGA Implementation of Bluetooth Low Energy Beacon-Based Indoor Positioning System
}

\author{
Chang Joo Song and Jeong Beom Kim
}

\begin{abstract}
In this paper, we described the field programmable gate array (FPGA) implementation of Bluetooth Low Energy (BLE) beacon-based indoor positioning system. Indoor positioning system relies on received signal strength indication (RSSI) from indoor wireless devices. The accuracy of indoor positioning system is easily affected by several signal interference. In order to correct for unstable RSSI values, Kalman filtering technique that has faster and lower error rate is adapted in this system. Also, we adopted the improved trilateration algorithm that has an error range of less than $0.5 \mathrm{~m}$. This system was implemented using Intel's FPGA design tools Quartus II on DE1-SoC board.
\end{abstract}

Keywords - Bluetooth low energy beacon, BLE, beacon, indoor positioning system, position-based service, Kalman filtering, improved trilateration algorithm

\section{INTRODUCTION}

In recent years, a localization technique, which is one of the core techniques in the wireless communications, has been rapidly developed with a variety of applications. As information technology industries rapidly develop, the various research themes such as location, home automation, and health care have been actively carried out. In particular, positioning technologies that provide services based on the object locations are receiving attention as a key technology in the internet of things (IoT) area. Conventional positioning services are now used in a wide range of areas as the global positioning system (GPS) technology in outdoor environments. However, receiving GPS signals is limited in confined spaces, such as inside buildings or underground. In addition, the typical measurement error range for GPS is $30 \mathrm{~m}$, which makes it unsuitable for measuring indoor locations. There are several location-based approaches to indoor posing system [1]-[2]. Indoor posing system is a meaningful method in application of position-based services. Recent research is actively carried out using Beacon based on Bluetooth 4.0 with the benefits of lightweight, low-cost and low-power conditions [3-6]. Bluetooth Low Energy (BLE) beacons-based IPS is a promising method for indoor posing system. However, beacon-based positioning is still very unstable and many developers are concerned because of its use of the value of received signal strength (RSSI).

In this paper, we achieved the device drive and fast data access by using the real-time and parallel processing of the field

Chang Joo Song is with the R\&D Division, Yura Corp., Seongnam, Republic of Korea.

Jeong Beom Kim is with the Department of Electronics Engineering, Kangwon National University, Chuncheon, Republic of Korea. programmable gate array (FPGA) built into the DE1-SoC board. Also, we implemented the signal filter algorithm and the improved trilateration algorithm by using hard process system (HPS). This paper is organized as follows; Section II provides Beacon and development environment. Section III describes RSSI filtering and coordinate calculation. Finally, we make conclusion in Section IV.

\section{BEACON AND DEVELOPMENT ENVIRONMENT}

\section{A. Beacon}

A beacon is an intentionally conspicuous device designed to attract attention to a specific location. BLE beacons are transmitter devices that broadcast signals, which can be picked up by nearby devices using Bluetooth. Most commonly they are used for advertising, and for informational purposes. These devices can take advantage of RSSI information together with an appropriate localization algorithm to pinpoint a user's position. This is particularly useful in indoor environments where GPS is no longer an option. Compared to other indoor posing system, BLE beacons is a low-cost, low-power option [7]. BLE beacon technology has developed during the last few years, as a means of sending position-based data to nearby users. Apple's iBeacon and Google's Eddystone are both examples of these small devices that can be attached to almost any surface, and then broadcast a signal which can be picked up by smartphones and other devices that utilize Bluetooth. Typically, BLE devices are used for advertising and informing users of nearby points of interest. However, using RSSI information, an application can estimate the distance to a beacon. Consequently, with the use of three or more beacons together with an effective algorithm, the user's position can be pinpointed [8].

BLE beacons are small devices that can be applied to almost any surface, and communicate messages to nearby devices using Bluetooth. The technology mainly finds its use within advertising, and informing smartphone users of nearby points of interests. A BLE beacon broadcasts small packets of data, with a certain interval. The maximum payload of a Bluetooth 4.2 packet is 257 bytes, which is not enough to embedded media content. Instead, a beacon simply broadcasts a unique ID and the application on the receiving device must recognize the beacon and perform relevant tasks. This is one-way communication, since beacons just broadcast signals and does not receive information [7]-[8]. BLE operates in the $2.4 \mathrm{GHz}$ license-free band (ISM band), which is the same frequency 
range as used in WiFi transceivers. This can cause some interference, resulting in packet loss, which can affect localization accuracy. On the other hand, a solution which utilizes both $\mathrm{WiFi}$ and Bluetooth simultaneously, gives better localization accuracy than using either technology individually. Beacon technology is a relatively low-cost solution, a single beacon generally ranging from 100-400 SEK, depending on how advanced the model. It is also a low-power device, as a beacon can have a lifespan of several years [5]. Availability is an advantage, since its features can be utilized by anyone with a smartphone. Broadcasting power can be adjusted on most beacons. This is a way to calibrate beacons for optimal distance estimation and positioning. For example, an estimated beacon has a power ranging between $4 \mathrm{dBm}$ and $-40 \mathrm{dBm}$, and the calibrated beacon ranges between $4 \mathrm{dBm}$ and $-30 \mathrm{dBm}$. Fig. 1 shows data packet structure of iBeacon.

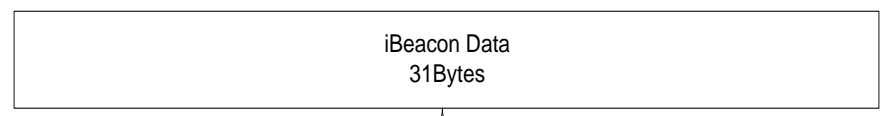

\begin{tabular}{|c|c|c|c|c|}
\hline $\begin{array}{c}\text { iBeacon Prefix } \\
\text { 9Bytes }\end{array}$ & $\begin{array}{c}\text { UUID } \\
\text { 16Bytes }\end{array}$ & $\begin{array}{c}\text { Major Number } \\
\text { 2Bytes }\end{array}$ & $\begin{array}{c}\text { Minor Number } \\
\text { 2Bytes }\end{array}$ & $\begin{array}{c}\text { TX Power } \\
\text { 1Bytes }\end{array}$ \\
\hline
\end{tabular}

\begin{tabular}{|c|c|c|c|c|}
\hline Adv Flags & Adv & Company & iBeacon & iBeacon \\
3B & Header & ID & Type & Length \\
(0x020106) & 2B & $2 \mathrm{~B}$ & $1 \mathrm{~B}$ & $1 \mathrm{~B}$ \\
& $(0 \times 1 \mathrm{AFF})$ & $(0 \times 004 \mathrm{C})$ & $(0 \times 02)$ & $(0 \times 15)$ \\
\hline
\end{tabular}

Fig. 1. Data packet structure of iBeacon

\section{B. System Configuration}

To implement the system, we used DE1-SoC board as the platform. The DE1-SoC board equipped with ARM Cortex-A processor integrated in HPS of the Altera Cyclone ${ }^{\circledR} \mathrm{V}$ system on a chip (SoC) FPGA. We used Quartus Prime 16.0 development tool that unified IDE and Eclipse Neon 3 development tool for embedded Linux programing. On Quartus Prime 16.0, we designed FPGA logic circuit using VHDL (VHSIC Hardware Description Language) and generated Handoff of HPS using Qsys. We executed Linux programming with the generated Handoff file and SSH (Secure Shell) communication in Eclipse.

Fig. 2 shows the proposed hardware block-diagram. FPGA logic circuit designed by VHDL connected with HPS and 32-bit bus. The hardware implemented by FPGA receives and selects Beacon data. The data packet broadcasted in 4 Beacon nodes are scanned by observer (BLE module). The scanned data is applied to FPGA logic circuit through UART (Universal Asynchronous Receiver/Transmitter) with synchronization rate of $9600 \mathrm{~Hz}$ Baud. UART implemented by DE1-SoC board stores the data word to buffer. The 8-bit data stored in buffer is ASCII code. When the first scan period of BLE module is finished, ASCII code of carriage return is transferred. Filtered Mac address and RSSI value of 4 fixed nodes in the stored string is transferred to HPS with 32-bit bus. The coordinate calculated in HPS is displayed in TFT LCD.

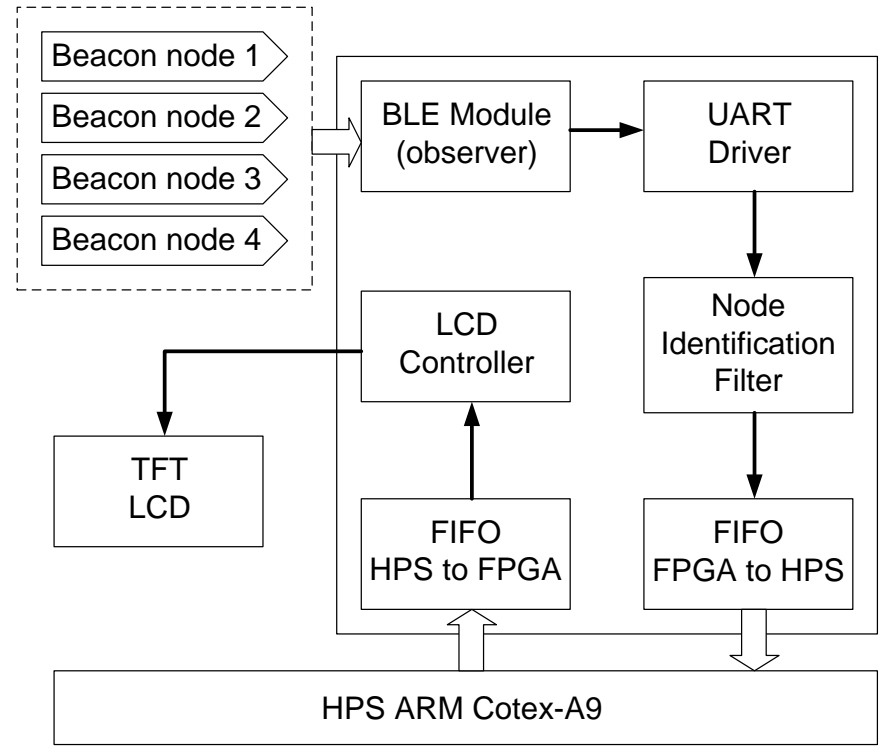

Fig. 2. Hardware block-diagram

Fig. 3 shows the software flowchart of the proposed system. To find the coordinate, the data from FPGA logic circuit is processed in HPS. The identified data with Mac address in FPGA logic circuit has the node numbers. The data in BLE module is randomly scanned. Therefore, beacon data with period of $12 \mathrm{~ms}$ should store in first-in first-out (FIFO). When each four identified ID is stored in array, the value is retuned. Completing this process, the stabilization of the data is carried out through RSSI rectifying algorithm. Through the improved trilateration algorithm, the accuracy of stabilized data is improved.

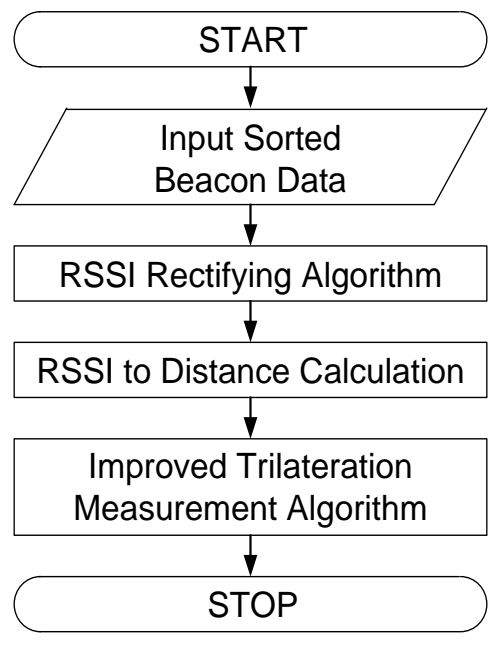

Fig. 3. Software flowchart

\section{RSSI FILTERING AND COORDINATE CALCULATION}

\section{A. RSSI Filtering}

RSSI, index of received signal strength, has $-99 \mathrm{dBm}$ to -35 $\mathrm{dBm}$. When the signal has strong strength, the signal has the high RSSI value. When beacon and the receiving module are close, RSSI value is high. We can calculate the distance 
between beacon and receiving module through the RSSI distance conversion formula. Table 1 shows experimental conditions. Experimental space is $4 \mathrm{~m} \times 4 \mathrm{~m}$, the signal only operates the measured beacon.

TABLE I: EXPERIMENTAL CONDITIONS

\begin{tabular}{ll}
\hline \hline Receiving mode & BoT- CLE110 \\
\hline Sending module (Beacon) & HM-10 \\
Signal transmission interval & $100 \mathrm{~ms}$ \\
Distance between Beacon and receiving module & $1 \mathrm{~m}$ \\
Sending signal strength & $-53 \mathrm{dBm}$ \\
\hline \hline
\end{tabular}

To estimate the distance from a beacon, a path-loss model is needed. In this implementation, we adopted the path-loss model [9],

$$
R S S I=-\left(10 n \log _{10} d+A\right)
$$

Where parameter $A$ is the absolute RSSI value represented by $\mathrm{dBm}$ at $1 \mathrm{~m}$ away from the beacon; $n$ is a parameter related to the signal propagation environment and $d$ is the distance from the beacon. In this implementation, we use the method proposed in [10] to estimate the parameters.

The path-loss model with pre-defined parameter in this implementation is

$$
R S S I=-\left(10 \times 2.5 \log _{10} d+30\right)
$$

Fig. 4 shows RSSI without filtering. We can find unstable signal distribution from Fig. 4.

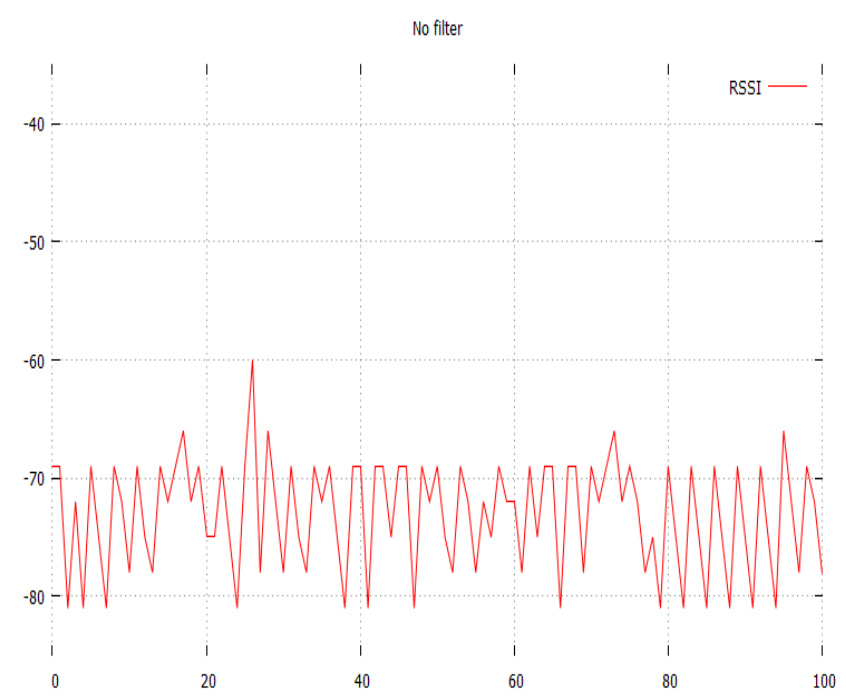

Fig. 4. RSSI without filtering

To stabilize the signal, we adopted Kalmam filtering. Kalman filtering is used for many applications including filtering noisy signals, generating non-observable states, and predicting future states. Filtering noisy signals is essential since many sensors have an output that is noisy too be used directly, and Kalman filtering lets is account for the uncertainty in the signal. Fig. 5 and 6 show Kalman filtering process and codes, respectively, where $\mathrm{K}$ is Kalman coefficient.

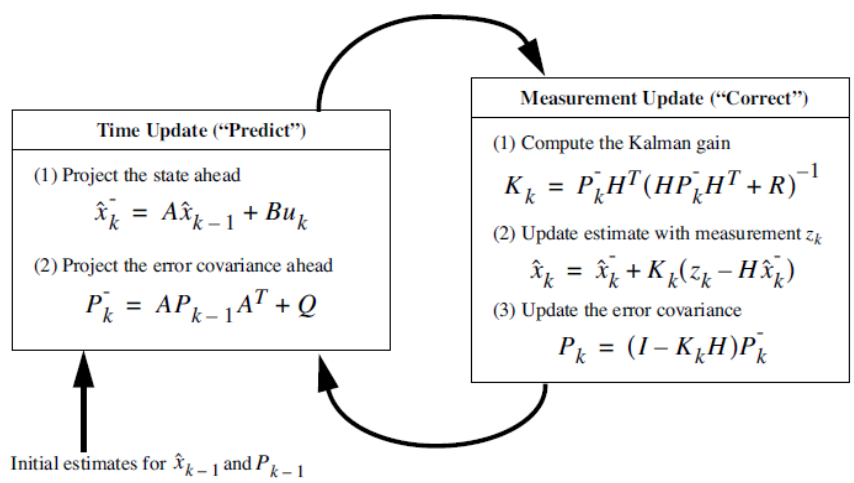

Fig. 5. Kalman filtering process

\begin{tabular}{l|l}
\hline 1 & $\mathrm{P}=\mathrm{P}+\mathrm{Q}$ \\
2 & $\mathrm{~K}=\mathrm{P} /(\mathrm{P}+\mathrm{R})$ \\
3 & $\mathrm{X}=\mathrm{K} * \mathrm{RSSI}+(1-\mathrm{K}) * \mathrm{X} ;$ \\
4 & $\mathrm{P}=(1-\mathrm{K}) * \mathrm{P} ;$ \\
\hline & Fig. 6. Kalman filtering code
\end{tabular}

Initially, we set $\mathrm{P}$ to $1.0, \mathrm{Q}$ to 0.001 , and $\mathrm{R}$ to 0.25 . By prediction and update process, error is reduced. Fig. 7 shows RSSI with filtering.

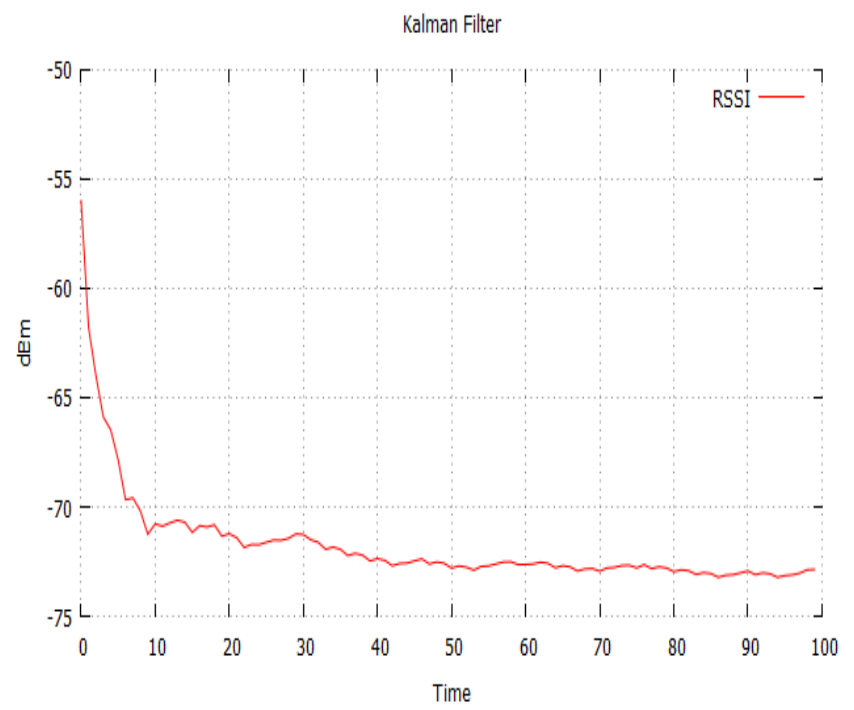

Fig. 7. RSSI with filtering

\section{B. Coordinate calculation}

Trilateration algorithm is widely used algorithm for recognizing location. However previous trilateration algorithm has some errors, exact coordinate calculation is difficult. To reduce error and to improve the accuracy, we adopted the improved trilateration algorithm. Fig. 8 shows the calculation of improved trilateration algorithm. 


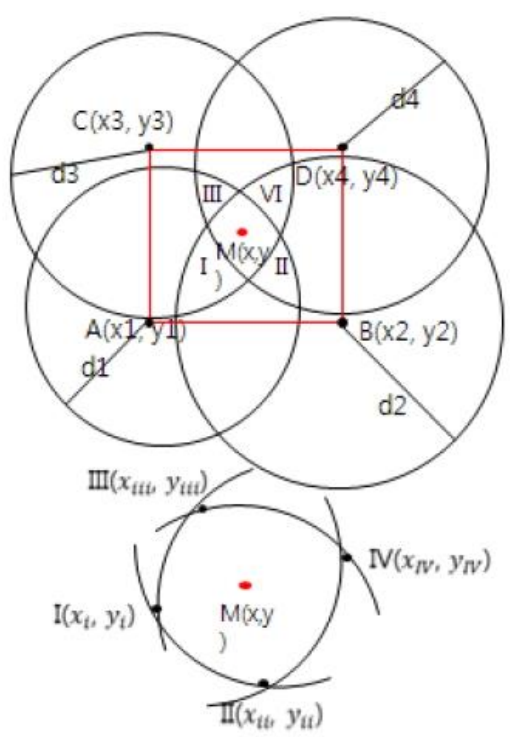

Fig. 8. Calculation of improved trilateration algorithm

This algorithm is set up as a zone with nine beacons and requires four coordinates. Due to the nature of the wireless signal, the accuracy decreases with the distance. Therefore, as a first step, we use the nine beacon signals to determine which zone the user is in, and then use four beacon signals in that zone to obtain the coordinates. The next step is to apply the improved trilateration algorithm to obtain the coordinates.

We can calculate the distance with Eq. (3) and modify Eq. (4). Finally, we can calculate the coordinates with Eq. (5).

$$
\begin{aligned}
& d_{1}^{2}=\left(x_{i}-x_{1}\right)^{2}+\left(y-y_{1}\right)^{2} \\
& d_{2}^{2}=\left(x_{i}-x_{2}\right)^{2}+\left(y-y_{2}\right)^{2} \\
& x_{i}=\frac{d_{1}^{2}-d_{2}^{2}+x_{2}^{2}-x_{1}^{2}}{2\left(x_{2}-x_{1}\right)} \\
& x=\frac{x_{i}+x_{i i}+x_{i i i}+x_{i v}}{4} \\
& y=\frac{y_{i}+y_{i i}+y_{i i i}+y_{i v}}{4}
\end{aligned}
$$

Fig. 9 shows the simulation results of the improved trilateration algorithm. In this work, we assume that there was a user in one of the four zones. We received one hundred RSSI data from four beacons installed at any given coordinate, and then simulated them using an improved trilateration algorithm. As simulation result, we can find that error range is reduced to less than $0.5 \mathrm{~m}$.

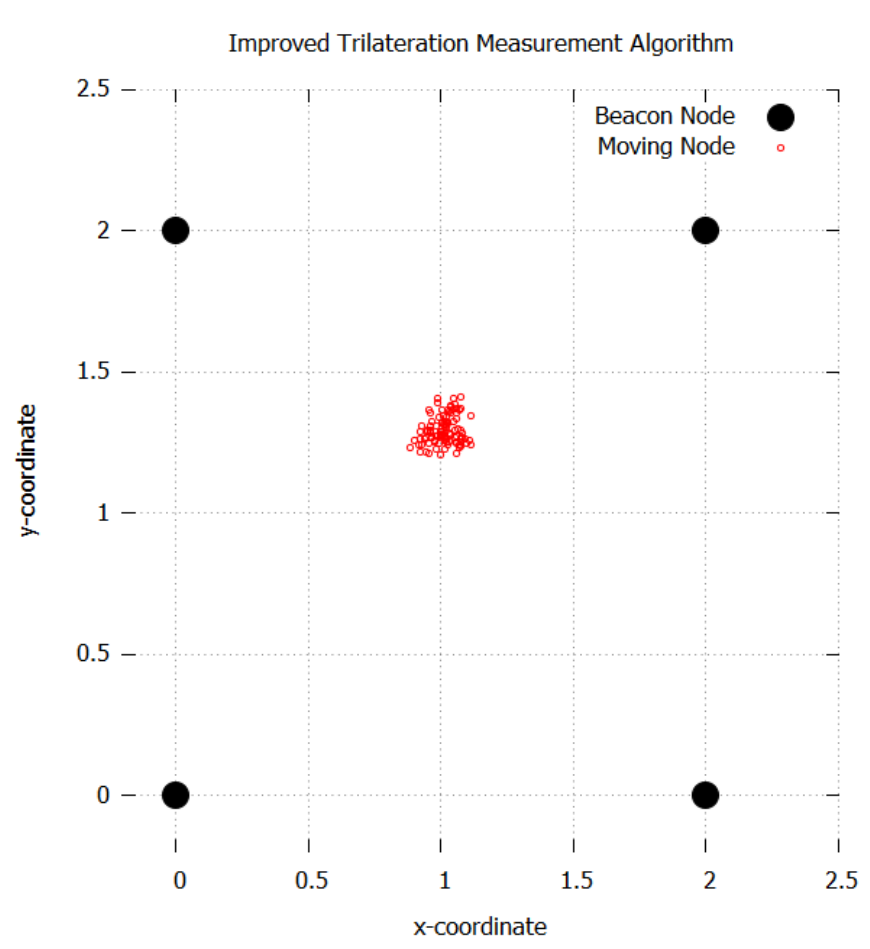

Fig. 9. Simulation results of the improved trilateration algorithm

\section{CONCLUSION}

We described FPGA implementation of BLE beacon-based indoor posing system. The accuracy of indoor posing system is easily affected by several signal interference. For the accuracy of the system, we adopted Kalman filtering technique and the improved trilateration algorithm. The improved trilateration algorithm reduces the error range. Intel's FPGA design tools Quartus II and DE1-SoC boards are used in this system. Future works are to enhance the reliability using secondary sensors such as WiFi, camera, and infrared sensors.

\section{ACKNOWLEDGMENT}

Authors thank the IDEC (IC Design Education Centre) program for its hardware and software assistance.

\section{REFERENCES}

[1] H. Liu, H. Darabi, P. Banerjee, J. Liu, "Survey of Wireless Indoor Positioning Techniques and Systems", IEEE Transactions on Systems, Man, and Cybernetics, vol. 37, no. 6, pp. 1067-1080, Nov. 2007. https://doi.org/10.1109/TSMCC.2007.905750

[2] A. Boukerche, H. Oliveira, E. F. Nakamura, "Localization systems for wireless sensor networks", IEEE Wireless Communications, pp. 6-12, Dec. 2007. https://doi.org/10.1109/MWC.2007.4407221

[3] R. Faragher, R. Harle, "Location Fingerprinting With Bluetooth Low Energy Beacons", IEEE Journal on Selected Areas in Communications, vol. 33, issue 11, pp. 2418-2428, Nov. 2015. https://doi.org/10.1109/JSAC.2015.2430281

[4] S. Aumumpai, K. Kodee, C. Prommak, K. Kaemarungsi, "Optimal Placement of Reference Nodes for Wireless Indoor Positioning Systems", Proceedings of the International Conference on Electrical Engineering/Electronics, Computer, Telecommunications and Information Technology (ECTI-CON), pp. 1-4, May 2014. https://doi.org/10.1109/ECTICon.2014.6839894 
[5] M. Golestanian, C Poellabauer, "Indoor Localization using Multi-Range Beaconing: Poster" MobiHoc 'Proceedings of the 17th ACM International Symposium on Mobile Ad Hoc Networking and Computing, p. 397-398, July 2016 https://doi.org/10.1145/2942358.2942414

[6] K. Bouchard, R Ramezani, Arjun, A. Naeim, "Evaluation of Bluetooth beacons behaviour", Proceedings of IEEE 7th Annual Ubiquitious Computing, Electronics \& Mobile Communication Conference (UEMCON), pp. 1-3, 2016. https://doi.org/10.1109/UEMCON.2016.7777846

[7] M. S. Arulampalam, S. Maskell, N. Gordon, and T. Clapp, "A tutorial on particle filters for online nonlinear/non-gaussian bayesian tracking," IEEE Transactions on Signal Processing, vol. 50, no. 2, pp. 174-188, Feb. 2002. https://doi.org/10.1109/78.978374

[8] S. Zhou, J. K. Pollard, "Position measurements using Bluetooth", IEEE Transactions on Consumer Electronics, vol. 52, issue 2, May 2006. https://doi.org/10.1109/TCE.2006.1649679

[9] Albowicz, J. "Receive position estimation in sensor network", Proceedings of the IEEE International Conference on Network Protocols, pp. 11-14, Nov. 2001.

[10] Wang, Y., Yang, X., Zhao, Y., and Liu, Y., Cuthbert, L. "Bluetooth positioning using RSSI and triangulation methods", Proceedings of the 2013 IEEE 10th Consumer Communications and Networking Conference (CCNC), pp. 837-842, Jan. 2013.
Chang Joo Song received B.S. degree in Electronic Engineering from Kangwon National University, Chucheon, Republic of Korea, in 2017. In 2017, he joined at R\&D Center, Yura Co. Ltd., Seongnam, Republic of Korea, where he is currently a senior engineer. His research interests include the design and FPGA implementation of positioning system.

Jeong Beom Kim received B.S. and M.S. degrees in Electronics Engineering from Inha University Incheon, Republic of Korea, in 1985 and 1987, respectively, and Ph.D. degree in Electronics and Electrical Engineering from Pohang University of Science and Technology (POSTECH), Pohang, Republic of Korea, in 1997. From 1987 to 1992, he worked at R\&D Center, Goldstar Semiconductor Co. Ltd., Seoul, Republic of Korea. From 1994 to 1998, he worked at System IC R\&D Center, Hyundai Electronics Co. Ltd., Icheon, Republic of Korea. From 1998 to 1999, he was with School of Electrical and Electronics Engineering, Chungbuk National University, Cheongju Republic of Korea. In 1999, he joined the Faculty of Electronics Engineering, Kangwon National University, Chuncheon, Republic of Korea, where he is currently a professor. His research interests include high-speed CMOS interface circuit, energy-efficient circuit, special purpose memory, and multi-valued logic circuit. He received the Best Paper Award from IKEEE and IASTED in 2005 and 2009, respectively. Prof. Kim is a member of IEEE and IEICE. 